\title{
Development and Maturation of the Autonomic Nervous System in Premature and Full-Term Infants Using Spectral Analysis of Heart Rate Fluctuations
}

\author{
UDI CHATOW, SHMUEL DAVIDSON, BRIAN L. REICHMAN, AND SOLANGE AKSELROD
}

Department of Neonatology, Beilinson Medical Center [S.D.] and Chaim-Sheba Medical Center [B.L.R.], and Department of Medical Physics, School of Physics [U.C., S.A.], Tel-Aviv University,

Tel-Aviv, Israel

The changes in the power spectra of heart rate (HR) fluctuations, in particular the total power (within $0.02-2.0 \mathrm{~Hz}$ ) and the power in the low- $(0.02-0.2 \mathrm{~Hz})$ and high- $(0.2-2.0 \mathrm{~Hz})$ frequency ranges, were computed from the ECG and respiratory signals of 59 premature and full-term infants. The objective of the study was to investigate the development and maturation of the autonomic nervous system from the first day of extrauterine life to several weeks of postnatal age. The study population was divided into four age groups. Group A: seven 1-d-old premature infants with gestational age of 34-35 wk. Group B: 28 premature infants 7-49 d old with a conceptional age of 34-35 wk. Group C: seven 1-d-old full-term infants of 39-41 wk gestation. Group D: six premature infants 35-97 d old with a conceptional age of 39-40 wk. Mean HR ( \pm SEM) of groups $C$ and D combined, i.e. $135 \pm 2 \mathrm{bpm}$, was significantly lower compared with groups $A$ and $\mathrm{B}$, i.e. $152 \pm 2(p<0.01)$. The mean $( \pm \mathrm{SEM})$ of the lowto high-frequency power ratio obtained from the HR power spectrum decreased progressively from $71 \pm 31$ in group $A$ to 34 \pm 8 in group $B, 16 \pm 3$ in group $C$, and $17 \pm 2$ in group $D$. The mean low to high ratio for the combined groups $C$ and $D, 17 \pm$ 1 , was significantly lower compared with the combined group A and $\mathrm{B}$, i.e. $44 \pm 9(p<0.01)$. The respiratory signals showed two types of breathing patterns: a single peak in the respiratory spectrum centered around the respiratory frequency (mature type, typically found in adults); and a second type showing two separate peaks, one centered around the respiratory frequency and the other at the much lower breath amplitude modulation frequency. In both respiratory modes, the HR power spectrum usually showed a dispersed, wide pattern of the power in the high-frequency range. The progressive decline in mean HR and in low- to high-power ratio indicates a decrease in sympathovagal balance with gestational and postnatal age. This maturation might be associated with a gradual focusing into a single respiratory peak, both in the respiratory and the HR power spectrum. (Pediatr Res 37: 294-302, 1995)
GA, gestational age
PA, postnatal age
CA, conceptional age $(\mathrm{CA}=\mathrm{GA}+\mathrm{PA})$
LFB, low-frequency band $(0.02-0.2 \mathrm{~Hz})$
HFB, high-frequency band $(0.2-2 \mathrm{~Hz})$
HR, heart rate
HRV, heart rate variability
ANS, autonomic nervous system
RSA, respiratory sinus arrythmia
BASA, breath amplitude sinus arrythmia

HRV is affected by numerous factors including blood pressure, temperature, respiration, biochemical influence of acid-base balance, state of oxygenation, ventilation, and psychologic parameters. It is a reflection of the cardiovascular control exerted by the ANS and a direct result of ANS functioning.

Using spectral analysis to investigate the frequency content of HR fluctuations, it has been shown that short-term fluctua-

Received August 2, 1993; accepted June 14, 1994.

Correspondence and reprint requests: S. Akselrod, Physics Department, Tel-Aviv University, Tel-Aviv, Israel. tions are concentrated in two principal frequency ranges (1-5) The high-frequency peak (HFB, above $0.2 \mathrm{~Hz}$ ) is centerer around the respiratory frequency and shifts with changes in thr respiratory rate $(1,3-5)$. The LFB of the power spectrun (below $0.2 \mathrm{~Hz}$ ) is composed of two not always distinct contri butions $(6,7)$. It contains the mid-frequency fluctuations be tween 0.09 and $0.15 \mathrm{~Hz}$, corresponding to what are sometime: called Mayer waves, which have been related to the barore ceptor reflex ( 7$)$. The other peak at the low-frequency end o the spectrum occurs typically within the $0.02-0.09 \mathrm{~Hz}$ rang and has been ascribed to thermoregulatory fluctuations an variations in vasomotor tone (6). The HFB reflects parasym 
pathetic activity, whereas the LFB is influenced by combined sympathetic and parasympathetic control (1). Their ratio is thus a reflection of the sympathovagal balance (8).

Previous animal research (9) suggests that the spectral approach may provide a description of the neurologic development, in particular certain aspects of autonomic function such as the sympathetic to parasympathetic balance. Assali et al. (10) studied the autonomic control of cardiovascular functions in a sheep model and followed the varying of maturation rates of both the sympathetic and parasympathetic systems. They found that the sympathetic nervous system matures earlier in fetal life than does parasympathetic control, whereas the cholinergic and adrenergic tones exerted on the resting $\mathrm{HR}$ at term are nearly balanced. This study also showed a pronounced increase of the parasympathetic tone after birth, whereas the adrenergic tone becomes less prevailing than in the fetus. Baldzer $e$ al . (11) studied a group of full-term neonates using this spectral approach and found that a difference in breathing pattern affects the HRV.

The aims of our work are 1) to study the changes in the HR power spectrum and the contributions of HRV to the various relevant frequency ranges in preterm and full-term infants from the first day of extrauterine life to several weeks PA to obtain information about autonomic maturation and, in particular, about sympathetic to parasympathetic balance; and 2) to study the mode of respiration observed in premature and full-term infants and its impinging effect on HRV.

The broad objectives of this investigation were to establish normative data for neonatal HRV, to determine the usefulness of spectral analysis as an aid to perinatal care, and to learn more about the development and maturation of the autonomic nervous system in newborns.

\section{METHODS}

Subjects. The study included 72 infants investigated during the period June 1988 to November 1990 . The infants were recorded during 20-min epochs. Some of them were followed and recorded during several such recording sessions, taken weeks apart as they matured.

All infants were healthy, requiring no oxygen administration and no medications except for xanthine therapy for apnea of prematurity in some subjects. Those who received xanthine showed a normal blood level of 7-12 $\mathrm{mg} / \mathrm{L}$. All had fewer than three episodes of apnea in a period of $24 \mathrm{~h}$, all of which resolved spontaneously. Their head ultrasonogram did not demonstrate any intraventricular hemorrhage.

The recordings started at least $24 \mathrm{~h}$ after birth, when the newborns had completed adaptation to extrauterine life successfully. The ECG and respiration recordings were performed in the neonatal intensive care unit and in the regular nursery using ECG electrodes attached in the standard three-lead manner. The full-term infants remained in their own cribs, whereas the premature infants' recordings were made in their incubators, which were adjusted to provide skin or air temperature according to standard criteria. Only those recordings made when the infants were either sleeping or awake but quiet were included in the analysis. No attempt was made to distinguish active from quiet sleep. Informed parental consent was obtained for each infant before the recording.

ECG recording and $H R$ spectral analysis. The ECG was measured with a Mennen Medical 740 neonatal monitor (Mennen Medical Ltd., Science Park, Rehovot, Israel). The analog ECG signals were recorded on a Hewlett-Packard 3968A FM magnetic tape recorder (Hewlett-Packard Co., Palo Alto, CA) at a tape speed of $15 / 16$ inch per second.

Spectral analysis of fluctuations in HR was performed offline on 256-s-long stationary segments of the ECG. These segments were sampled at a frequency of $500 \mathrm{~Hz}$ and analyzed using a PDP 11-23 minicomputer (Digital) with an analog to digital converter. The R-waves were detected, and the values of $R R$ intervals were saved. The number of RR intervals within a time window of 0.5 -s width were used to provide a low pass-filtered series of instantaneous HR values, sampled at equal time intervals according to a previously described algorithm. A smooth time series of instantaneous HR sampled at frequency $\mathrm{f}=4 \mathrm{~Hz}$ was thus created. Stationarity of these HR traces was established by subdividing the traces into four subtraces and assessing that the mean $H R$ value and the variance of the HR fluctuations computed for each subtrace were not significantly different.

Next, the HR power spectrum was estimated based on the computation of a fast Fourier transform of the HR time series. This approach was chosen because relatively long ( $256 \mathrm{~s})$ and steady traces are considered (2). The power spectrum was smoothed with a five-point Hanning window and normalized by mean HR squared (1). The HR power spectrum thus obtained is presented in units of $\mathrm{Hz}^{-1}$, and the integrals over specific frequency bands are thus unitless (1).

Frequency ranges of interest. Neonates exhibit HRV fluctuations in a wide range of frequencies as shown in a typical power spectrum (Fig. 1). We investigated the entire bandwidth up to $2 \mathrm{~Hz}$ except for the very low $(0-0.02 \mathrm{~Hz})$ band, which was discarded to avoid the possible contribution of slow trend artifacts. The examined $0.02-2.0 \mathrm{~Hz}$ range was divided into several frequency bands, the main divisions being the LFB $(0.02-0.2 \mathrm{~Hz})$ and the HFB $(0.2-2.0 \mathrm{~Hz})$.

The HFB contains primarily the reflection of the respiratory activity (1, 3-5). The LFB includes at least two contributions $(1,3-8)$, the thermoregulatory fluctuations tone (below 0.09 $\mathrm{Hz}$ ) and the baroreceptor control fluctuations (typically occurring between 0.09 and $0.15 \mathrm{~Hz}$ ). The LFB also possibly may contain fluctuations due to breath amplitude modulation (12).

Respiratory data analysis. Whenever available for research, a Mennen Medical 744 neonatal monitor was used, also providing us with a respiratory recording. In those cases that allow examination of both HR and respiration, both signals were recorded and digitized simultaneously.

The respiratory signal was sampled at $4 \mathrm{~Hz}$, after analog low-pass filtering. The respiratory power spectrum, computed similarly to the HR signal, provided the exact frequency limits of the respiratory frequency band, which did not exceed the upper limit of $50-60$ breaths per minute. The power spectra of both $\mathrm{HR}$ and respiratory signals were used to ascertain whether common frequency patterns occur in these signals. 

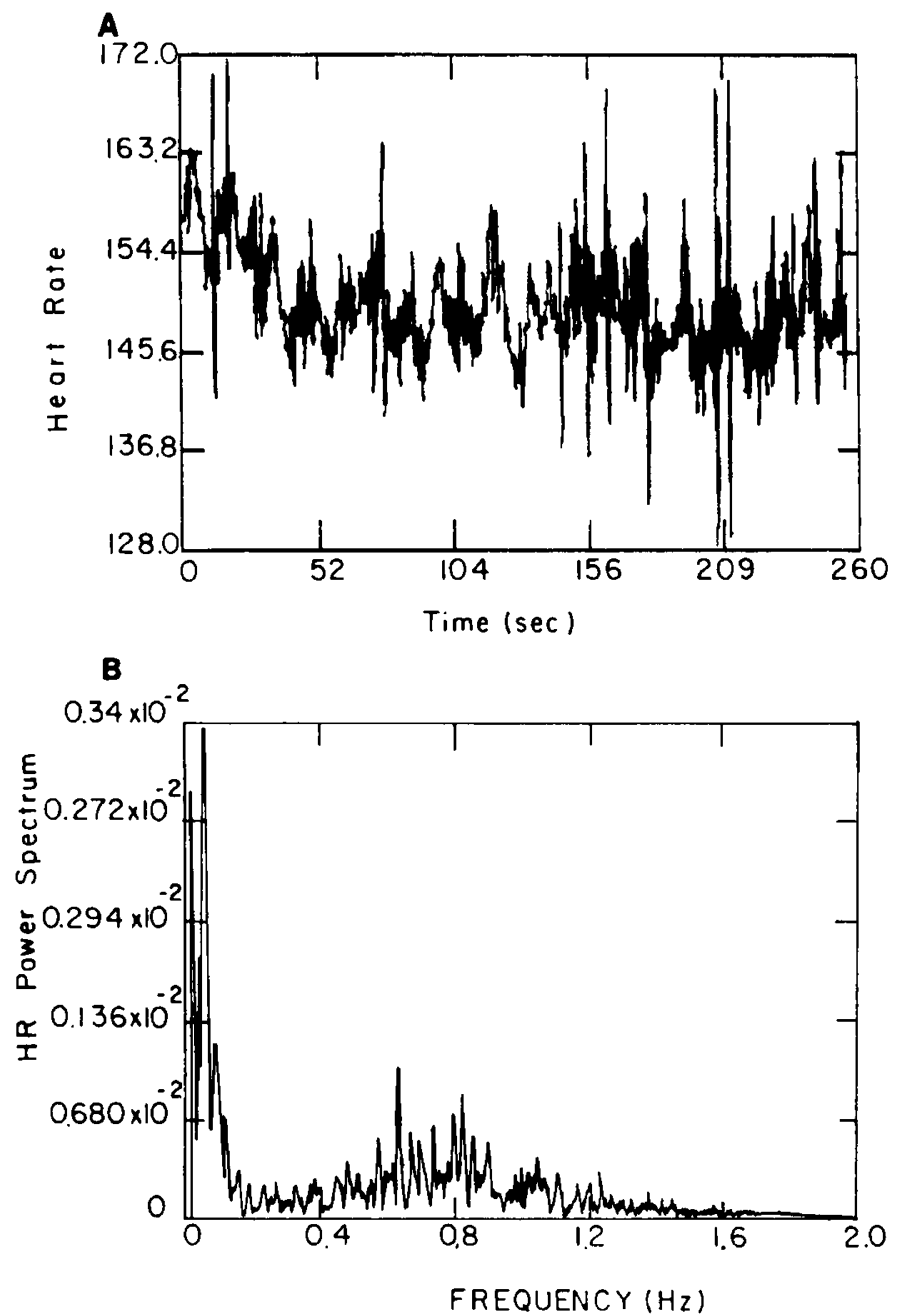

Figure 1. $A$, An example of a typical HR time series for a newborn (group D, number 43 ). $B$, Corresponding power spectrum (in units of $1 / \mathrm{Hz}$ ) of $\mathrm{HR}$ fluctuations, displaying regions of power both in the HFB $(0.2-2.0 \mathrm{~Hz})$ and in the LFB $(0.02-0.2 \mathrm{~Hz})$.

Statistical analysis. Differences between the mean values for the various age groups were analyzed by the $t$ test; $p<0.05$ was accepted as statistically significant. Linear regression was performed to achieve a linear fit and find the coefficient relating the low to high ratio as well as the HFB power to the postnatal age.

\section{RESULTS}

Age distribution of infants. From the 72 infants with 110 recording sessions, 59 infants with 85 recordings were analyzed and included in the study. Twenty-five recording sessions of 13 infants were excluded for bad quality signals with many artifacts that did not allow adequate analysis.

Clinical data for the investigated population are shown in Table 1. During the analysis of the recordings, the population was grouped according to GA and PA (Table 2): group A: seven preterm infants, GA 34-35 wk, recorded $24 \mathrm{~h}$ after birth; group B: Twenty-eight preterm infants born at 27-34 wk GA with a PA of 1-7 wk for a $\mathrm{CA}(\mathrm{CA}=\mathrm{GA}+\mathrm{PA})$ similar to that of group A (33-35 wk); group C: Seven full-term infants, GA 39-41 wk, recorded $24 \mathrm{~h}$ after birth; and group D: Six preterm
Table 1. Clinical data

\begin{tabular}{ccccc}
\hline $\begin{array}{c}\text { GA } \\
(\text { wk })\end{array}$ & $\begin{array}{c}\text { No. in } \\
\text { group }\end{array}$ & $\begin{array}{c}\text { Birth weight } \\
\text { mean }(\mathrm{g})\end{array}$ & $\begin{array}{c}\text { Birth weight } \\
\text { SD }(\mathrm{g})\end{array}$ & $\begin{array}{c}\text { Mean Apgar } \\
\text { score }\end{array}$ \\
\hline 26 & 3 & 695 & 90 & 6 \\
27 & 4 & 950 & 160 & 8.25 \\
28 & 2 & 1280 & 170 & 7 \\
29 & 3 & 1210 & 120 & 9 \\
30 & 5 & 1290 & 110 & 8 \\
31 & 6 & 1337 & 105 & 8.3 \\
32 & 4 & 1525 & 350 & 8.5 \\
33 & 3 & 2023 & 377 & 7.7 \\
34 & 11 & 1920 & 170 & 8.7 \\
35 & 6 & 1950 & 126 & 8 \\
36 & 5 & 2190 & 575 & 8.5 \\
39 & 1 & 4100 & 0 & 9 \\
40 & 4 & 3400 & 76 & 9.25 \\
41 & 2 & 3510 & 152 & 9 \\
\hline
\end{tabular}

infants born at 27-34 wk GA with a PA of 5-14 wk for a CA similar to that of group C (39-41 wk).

An additional group of infants $(n=11)$ that did not belong to the four age groups were not included in the study. Their data are not displayed in Table 2; however, the data are available upon request.

Mean HR. First, we present the mean HR values because this is the most direct, basic, and well-accepted parameter that we can extract from our data. It is the first approximation of what can be learned from HR about the development of autonomic control in premature versus full-term babies. The results of the spectral analysis go one step further to a secondorder approximation of what HR can teach us by focusing on its fluctuations.

Comparing the mean HR ( \pm SEM) among the age groups (Fig. 2, Table 2), we find the highest HR in group B with 156 $\pm 2 \mathrm{bpm}$ followed by group D with an HR of $146 \pm 7.5 \mathrm{bpm}$; both groups had a PA of more than 1 wk, and the 1-d-old neonates in groups $\mathrm{A}$ and $\mathrm{C}$ showed a lower mean HR of 133 \pm 4 and $124 \pm 4 \mathrm{bpm}$, respectively (Fig. 2). As shown in Figure 2, $t$ test comparison of mean HR reveals a statistically

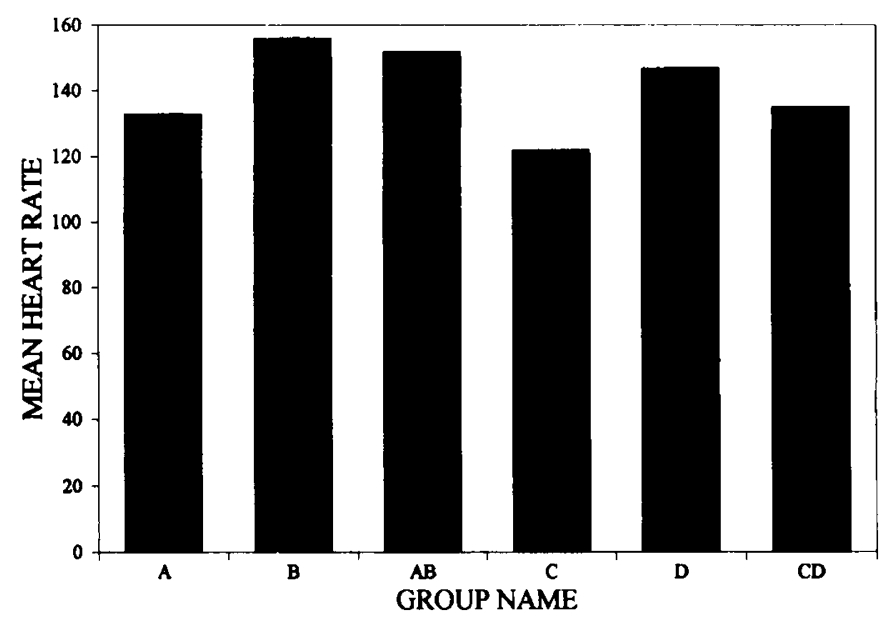

Figure 2. Mean HR of the four age groups and the combined age groups; their SEM are presented in Table 2. A significant difference $(p<0.01)$ is observed between groups $A$ and $B$, groups $C$ and $D$, and the combined groups $A B$ and $C D$. 
significant difference between groups $\mathrm{A}$ and $\mathrm{B}(p<.001)$ as well as between groups $\mathrm{C}$ and $\mathrm{D}(p=0.02)$.

The combined mean HR ( \pm SEM) found for the combined group $\mathrm{AB}$ was $152 \pm 2 \mathrm{bpm}$, significantly different from the CD group, which had $135 \pm 2 \mathrm{bpm}(p<0.01)$.

The general conclusion is that HR decreases and thus vagal activity becomes more predominant with $\mathrm{GA}\left(\mathrm{HR}_{\mathrm{AB}}>\mathrm{HR}_{\mathrm{CD}}\right)$. Also, vagal control seems to develop better in the womb, as indicated by the comparison of mean HR within groups having the same CA in which $\mathrm{HR}$ is higher for higher postnatal ages $\left(\mathrm{HR}_{\mathrm{A}}<\mathrm{HR}_{\mathrm{B}}\right.$ and $\left.\mathrm{HR}_{\mathrm{C}}<\mathrm{HR}_{\mathrm{D}}\right)$.

Neonatal variability. Two typical modes of neonatal HR power spectra are observed.

Figure $1 A$, is an example of a neonatal HR time series with Figure $1 B$ showing the corresponding HR power spectrum. This spectrum is typical for one of the two main modes found in neonatal power spectra. In this mode we find a wide but clear respiratory peak. The high-frequency respiratory variations in HR are expressed by the peak centered at $0.8 \mathrm{~Hz}$. The high-frequency power is not limited here to a narrow and sharp peak appearing in the HR power spectrum as has been found in adults, but rather it is a wider band of dispersive type spreading almost throughout the entire HFB. When considering the HR power spectrum from 256-s traces, it is rare to find in newborns, and particularly in premature neonates, a narrow, welldefined respiratory peak such as in adults; actually, in our study, none were found.

Figure $3 A$ shows another example of an HR time series of $256 \mathrm{~s}$. The instantaneous HR varies periodically with oscillations 10 and $20 \mathrm{~s}$ long. Figure $3 B$ displays the corresponding power spectrum. As can be seen, there is not much power above $0.2 \mathrm{~Hz}$ in the HFB, which in adults usually displays the reflection of the respiratory activity. The LFB shows two typical peaks at $0.04 \mathrm{~Hz}$ and $0.11 \mathrm{~Hz}$. The high-frequency range displays an undefined, wide, and very small respiratory peak that is not even apparent in the HR power spectrum.

Because the two recording sessions presented in Figures 1 and 3 were from the same infant at a time interval of $1 \mathrm{wk}$, a comparison between their spectra emphasizes the problem of nonuniformity and variability in neonatal HR power spectra.

Table 2 displays the spectral information for all subjects in the four age groups and emphasizes the considerable changes that may occur in the HR power spectrum in the different frequency bands when comparing subjects, both within and across the various groups. Considering the LFB $(0.02-0.2 \mathrm{~Hz})$, in which most of the power is concentrated for neonates, we find that the mean power for group C (full-term infants) is 0.33 $\pm 0.1 \times 10^{-3}$ (mean \pm SEM), displaying a very large variability within the group. A similar variability is found in the power in other spectral bands and in the total power, when comparing within the group. In Table 2, we thus showed, for each infant, the percentage column next to the absolute power in every band, representing the power in the specific band as a proportion of the total power. The mean percentage power in the LFB for group C is $93.4 \pm 1 \%$ (mean \pm SEM), presenting a much lower variability.

Group D, including preterm neonates at full-term CA, resembles group $\mathrm{C}$. Although on an absolute scale group $\mathrm{D}$ has
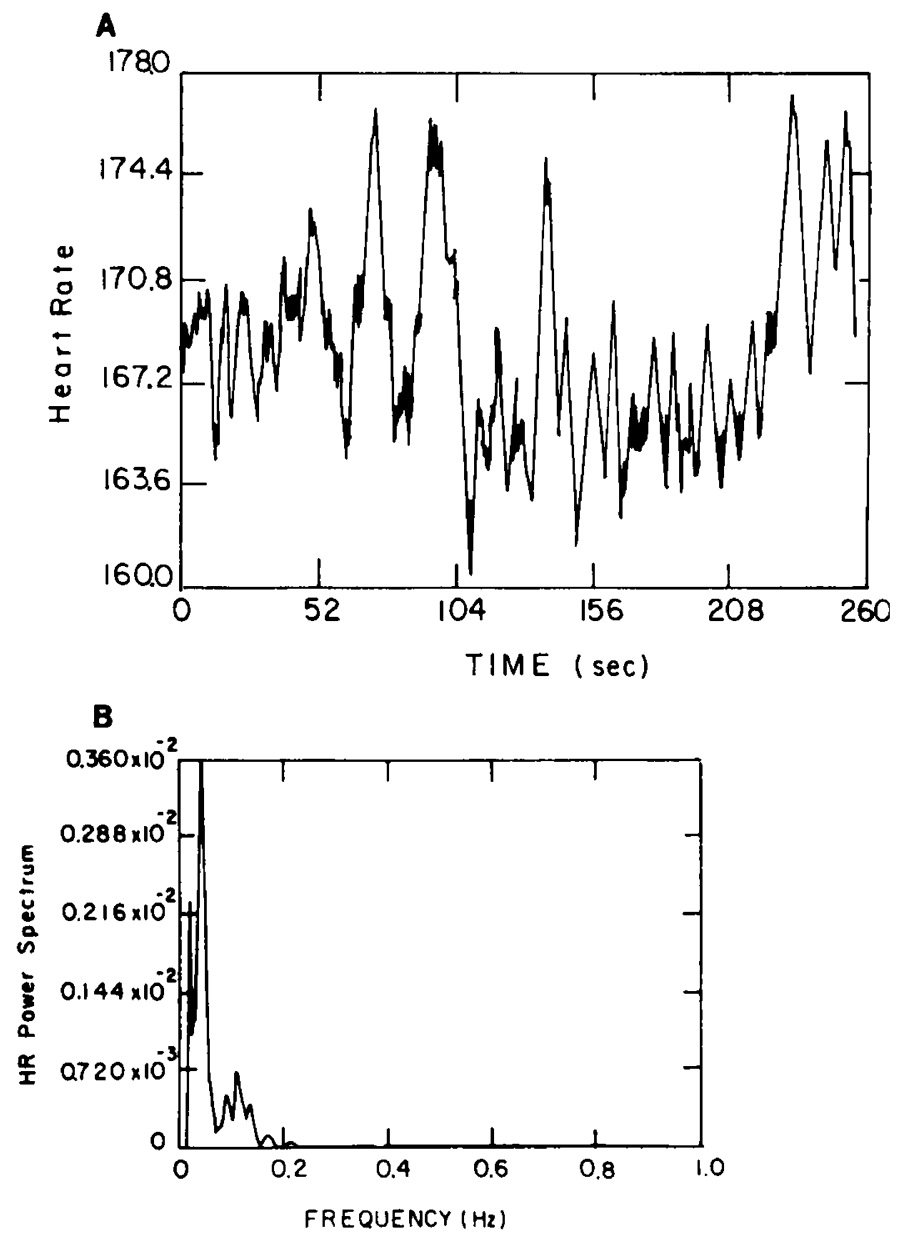

Figure 3. $A$, An example of another HR time series for the same infant as in Figure $1 A$ (group $\mathrm{D}$, number 43 ). $B$, Corresponding power spectrum of $\mathrm{HR}$ fluctuations, displaying the main region of power to be in the LFB (0.02-0.2 $\mathrm{Hz}$ ), where several peaks appear.

slightly more than twice the total power (compared with group C), the mean LFB percentage reaches $94.2 \pm 0.6 \%$ (mean \pm SEM). Again the SEM of the mean total power is very large, possibly hinting at the various modes of CNS activity through which the infant may switch.

This wide variability agrees with the large changes in power that also are found in the same infant during different recording sessions (Figs. $1 B$ and $3 B$ ).

Age dependence or maturation. A clear dependence of neonatal autonomic control on CA, GA, or both is found in this study, as displayed by the changes in sympathovagal balance expressed by the ratio of LFB to HFB power. Table 2 gives the average low- to high-power ratio for each age group with the SEM.

Group A, which includes 1-d-old preterm infants with a GA of 34 and $35 \mathrm{wk}$, has an average ratio of $71 \pm 31$ (mean \pm SEM). Group B, 33-35 wk CA with PA in the range of 1-7 wk, shows a mean low to high ratio of $34 \pm 8$. Group $C$, full-term infants recorded $24 \mathrm{~h}$ after birth, presents a ratio of $16 \pm 3$. The mean ratio for group $\mathrm{C}$ is obtained by omitting the two extreme ratios, marked "-" near their name in Table 2; a similar general trend was observed when including these two excluded infants. Group D, 39-41 wk CA, reaches a similar ratio of 17 \pm 2 , although its average HR was higher than that for group C. 
Table 2. Results of spectral analysis for all subjects and averaged over groups

\begin{tabular}{|c|c|c|c|c|c|c|c|c|c|c|c|c|}
\hline Name & $\begin{array}{l}\text { GA } \\
\text { (wk) }\end{array}$ & $\begin{array}{l}\text { PA } \\
\text { (d) }\end{array}$ & $\begin{array}{c}\text { CA } \\
\text { (wk) }\end{array}$ & $\begin{array}{c}\text { Mean } \\
\text { HR }\end{array}$ & $\begin{array}{c}\text { Total } \\
\text { power }\end{array}$ & $\begin{array}{c}\text { Low } \\
(0.02-0.2)\end{array}$ & $\begin{array}{l}\text { High } \\
(0.2-2)\end{array}$ & $\begin{array}{c}\text { Low to } \\
\text { high ratio }\end{array}$ & $\begin{array}{c}0.05-0.09 \\
\mathrm{~Hz}(\%)\end{array}$ & $\begin{array}{c}0.09-0.15 \\
\mathrm{~Hz}(\%)\end{array}$ & $\begin{array}{l}\text { Low } \\
(\%)\end{array}$ & $\begin{array}{l}\text { High } \\
(\%)\end{array}$ \\
\hline \multicolumn{13}{|l|}{ Group A } \\
\hline 1 & 34 & 1 & 34 & 143 & 0.150 & 0.111 & 0.039 & 2.9 & 35.9 & 17.2 & 74.0 & 26.0 \\
\hline 3 & 34 & 1 & 34 & 114 & 0.456 & 0.454 & 0.002 & 227.0 & 60.1 & 8.4 & 99.6 & 0.4 \\
\hline 4 & 34 & 1 & 34 & 127 & 0.482 & 0.420 & 0.0614 & 6.8 & 41.1 & 12.3 & 87.3 & 12.7 \\
\hline 5 & 35 & 1 & 35 & 133 & 0.914 & 0.482 & 0.4317 & 1.1 & 23.0 & 10.2 & 52.7 & 47.3 \\
\hline Mean & 34.4 & 1 & 34 & 133 & 0.40 & 0.32 & 0.08 & 71.4 & 44.8 & 17.0 & 86.8 & 13.2 \\
\hline SEM & 0.2 & 0.0 & 0.2 & 4.0 & 0.1 & 0.1 & 0.1 & 30.9 & 4.4 & 2.7 & 6.2 & 6.2 \\
\hline \multicolumn{13}{|l|}{ Group B } \\
\hline 8 & 34 & 7 & 35 & 149 & 0.210 & 0.208 & 0.0016 & 130.0 & 49.5 & 19.8 & 99.2 & 0.8 \\
\hline 9 & 32 & 8 & 33 & 161 & 0.049 & 0.048 & 0.001 & 48.2 & 50.1 & 32.8 & 98.1 & 1.9 \\
\hline 14 & 33 & 10 & 34 & 146 & 0.201 & 0.173 & 0.028 & 6.2 & 34.3 & 11.9 & 86.1 & 5.0 \\
\hline 15 & 34 & 11 & 35 & 153 & 0.147 & 0.138 & 0.009 & 15.3 & 42.2 & 30.6 & 93.9 & 3.4 \\
\hline 16 & 33 & 12 & 35 & 146 & 0.318 & 0.315 & 0.0028 & 112.5 & 52.2 & 26.6 & 99.1 & 0.9 \\
\hline 17 & 31 & 13 & 33 & 149 & 0.783 & 0.679 & 0.104 & 6.5 & 21.8 & 18.4 & 86.7 & 6.9 \\
\hline 18 & 32 & 14 & 34 & 154 & 0.160 & 0.158 & 0.00257 & 61.4 & 53.0 & 25.8 & 98.4 & 1.6 \\
\hline 19 & 31 & 16 & 33 & 158 & 0.088 & 0.084 & 0.00336 & 25.1 & 54.2 & 16.4 & 96.2 & 3.8 \\
\hline 20 & 31 & 18 & 34 & 148 & 0.246 & 0.242 & 0.00359 & 67.4 & 33.9 & 14.9 & 98.5 & 1.5 \\
\hline 21 & 30 & 19 & 33 & 150 & 0.181 & 0.168 & 0.0131 & 12.8 & 48.5 & 15.8 & 92.8 & 7.2 \\
\hline 22 & 32 & 21 & 35 & 161 & 0.738 & 0.706 & 0.03233 & 21.8 & 37.7 & 17.4 & 95.6 & 4.4 \\
\hline 23 & 32 & 21 & 35 & 147 & 0.726 & 0.614 & 0.112 & 5.5 & 14.2 & 13.9 & 84.6 & 21.1 \\
\hline 24 & 31 & 22 & 34 & 167 & 0.187 & 0.186 & 0.0012 & 154.8 & 49.3 & 16.8 & 99.4 & 0.6 \\
\hline 25 & 31 & 23 & 34 & 166 & 0.188 & 0.181 & 0.0067 & 27.0 & 46.8 & 18.8 & 96.4 & 3.6 \\
\hline 35 & 27 & 49 & 34 & 167 & 0.278 & 0.262 & 0.0163 & 16.1 & 50.0 & 19.8 & 94.1 & 5.9 \\
\hline Mean & 31.2 & 19.7 & 34.0 & 156.2 & 0.43 & 0.36 & 0.07 & 34.4 & 37.2 & 18.4 & 86.0 & 12.5 \\
\hline SEM & 0.3 & 1.8 & 0.2 & 1.8 & 0.07 & 0.06 & 0.02 & 8.4 & 2.8 & 1.4 & 3.3 & 3.1 \\
\hline \multicolumn{13}{|l|}{ Group C } \\
\hline 36 & 39 & 1 & 39 & 137 & 0.161 & 0.151 & 0.0102 & 14.8 & 37.5 & 15.6 & 93.7 & 6.3 \\
\hline $37-$ & 40 & 1 & 40 & 138 & 1.151 & 1.137 & 0.0148 & 76.8 & 50.2 & 24.6 & 98.7 & 1.3 \\
\hline 38 & 40 & 1 & 40 & 121 & 0.106 & 0.102 & 0.00388 & 26.4 & 59.7 & 19.0 & 96.3 & 3.7 \\
\hline 39 & 40 & 1 & 40 & 112 & 0.763 & 0.694 & 0.0684 & 10.1 & 30.5 & 28.9 & 91.0 & 9.0 \\
\hline 40 & 40 & 1 & 40 & 134 & 0.172 & 0.164 & 0.00822 & 19.9 & 32.7 & 28.7 & 95.2 & 4.8 \\
\hline 41 & 41 & 1 & 41 & 116 & 0.580 & 0.527 & 0.0525 & 10.0 & 33.4 & 26.2 & 90.9 & 9.1 \\
\hline $42-$ & 41 & 1 & 41 & 96 & 0.570 & 0.389 & 0.1809 & 2.2 & 35.9 & 16.2 & 68.3 & 31.7 \\
\hline Mean & 40.0 & 1.0 & 40.0 & 124.0 & 0.36 & 0.33 & 0.03 & 16.3 & 38.8 & 23.7 & 93.4 & 6.6 \\
\hline SEM & 0.3 & 0.0 & 0.3 & 4.4 & 0.12 & 0.11 & 0.01 & 2.8 & 4.8 & 2.4 & 1.0 & 1.0 \\
\hline \multicolumn{13}{|l|}{ Group D } \\
\hline $43-$ & 34 & 35 & 39 & 150 & 0.297 & 0.147 & 0.15 & 1.0 & 23.0 & 10.0 & 49.5 & 50.5 \\
\hline 44 & 34 & 42 & 40 & 169 & 0.114 & 0.108 & 0.0061 & 17.7 & 15.1 & 18.8 & 94.7 & 5.3 \\
\hline 45 & 32 & 49 & 39 & 164 & 0.245 & 0.234 & 0.01104 & 21.2 & 48.0 & 19.8 & 95.5 & 4.5 \\
\hline 46 & 27 & 94 & 39 & 137 & 0.918 & 0.839 & 0.0783 & 10.7 & 24.9 & 33.1 & 91.5 & 8.5 \\
\hline 47 & 27 & 94 & 39 & 127 & 0.364 & 0.344 & 0.02 & 17.2 & 35.0 & 25.3 & 94.5 & 5.5 \\
\hline 48 & 27 & 97 & 40 & 135 & 2.054 & 1.949 & 0.105 & 18.6 & 28.4 & 22.3 & 94.9 & 5.1 \\
\hline Mean & 29.4 & 75.2 & 39.4 & 146.4 & 0.74 & 0.69 & 0.04 & 17.1 & 30.3 & 23.9 & 94.2 & 5.8 \\
\hline SEM & 1.3 & 10.9 & 0.2 & 7.5 & 0.32 & 0.30 & 0.02 & 1.5 & 4.9 & 2.3 & 0.6 & 0.6 \\
\hline
\end{tabular}


Combining age groups $\mathrm{A}$ and $\mathrm{B}(\mathrm{CA}=33-35 \mathrm{wk})$ and $\mathrm{C}$ and $D(C A=39-41 \mathrm{wk})$, the new mean values obtained for the low to high band ratio are $44 \pm 9$ for the $\mathrm{AB}$ group, whereas the ratio for the combined $\mathrm{CD}$ group is $17 \pm 1$. Thus a significant $p<0.01$ is achieved for the low to high ratio comparison between groups $\mathrm{AB}$ and $\mathrm{CD}$. Figure $4 A$ displays the trend observed in the form of a bar graph.

Taking the largest age group, $\mathrm{B}(\mathrm{CA}=33-35)$, and plotting (Fig. $4 B$ ) the low- to high-power ratio as a function of PA within the group, we obtain a statistically nonsignificant
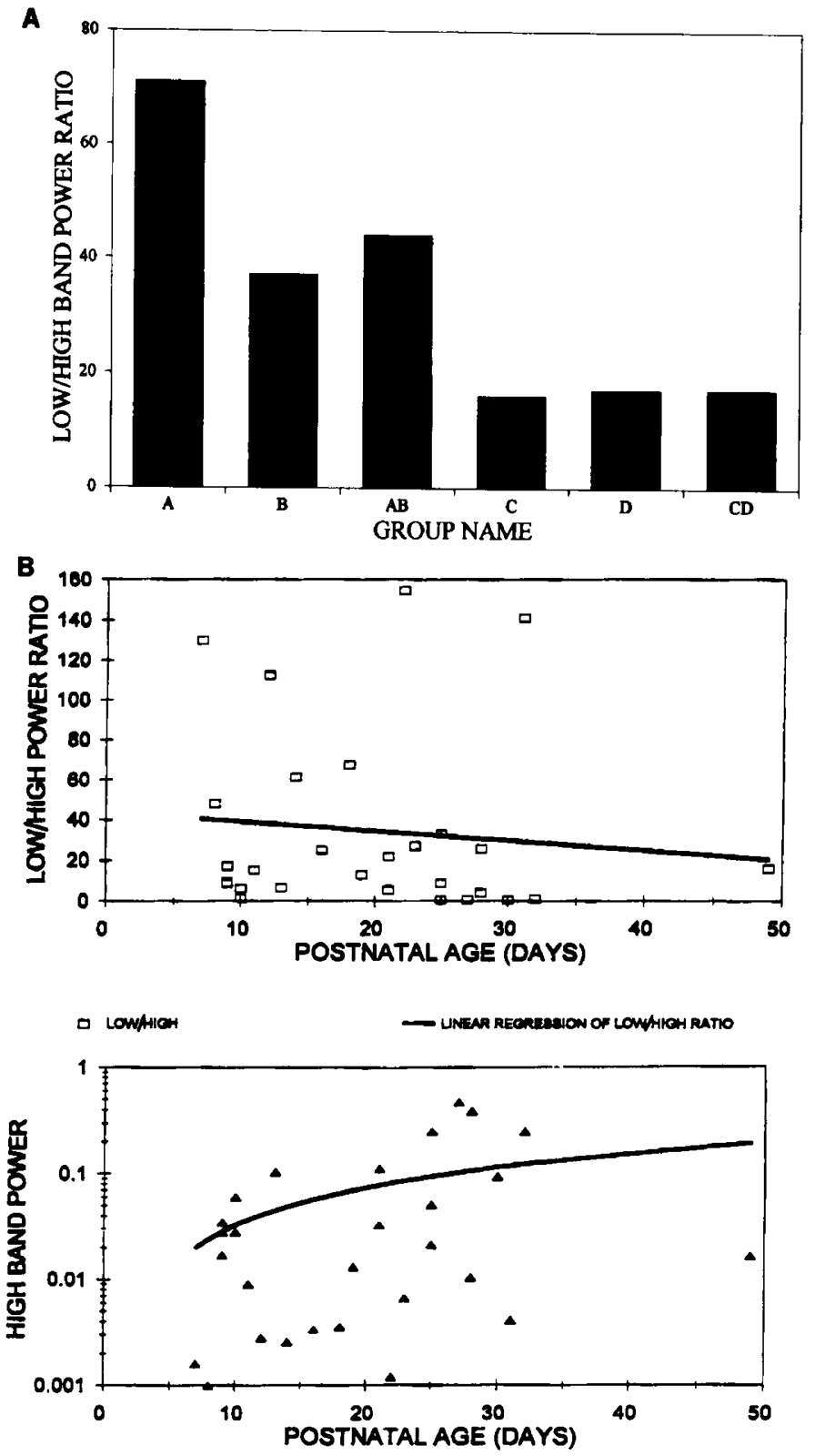

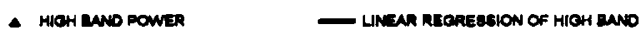

Figure 4. $A$, Mean LFB to HFB power ratio for the four age groups and the combined age groups; their SEM are indicated in Table 2. Groups AB and CD display a significant difference $(p<0.01)$. B. Upper panel, The low to high ratio (linear scale) of group B preterm infants, displayed as a function of PA, together with its respective regression line. Lower panel, the HFB power spectrum (logarithmic scale) of group B preterm infants, displayed as a function of PA, together with its respective regression line. negative coefficient $(-0.57)$ for the linear fit (SEM $=1.08$ and $\left.r^{2}=0.01\right)$. The second graph, shown in Figure $4 B$, displays the HFB power as a function of PA. The coefficient of this linear regression is 0.0063 (SEM $=0.0026, r^{2}=0.18$, and $p<0.05$ ), indicating a slight and gradual increase in the HFB or in parasympathetic activity as PA progresses. Note that the HFB power in Figure $4 B$ is plotted on a logarithmic scale because of the wide range of values.

Respiratory influence on HRV. Important additional information was obtained from the respiratory signal when recorded with the ECG and analyzed simultaneously with HR. Inspecting the respiratory time series and respiratory power spectra, we found two main types: 1) a single peak in the spectrum centered around the respiratory frequency, and 2) two separate peaks, the first centered around the respiratory frequency (RSA) and the second around the frequency of the breath amplitude modulation (BASA) (12).

The first type seems similar to an adult (mature) respiratory power spectrum except for the tendency of the respiratory peak to be wider in neonates, especially in premature infants. Figure $5 B$ shows such a single-peak respiratory spectrum. Figure $5 A$ shows the corresponding HR power spectrum for this neonate: it displays a wide respiratory peak in the HFB. The corresponding respiratory spectrum (Fig. $5 B$ ) shows a fairly sharp peak at

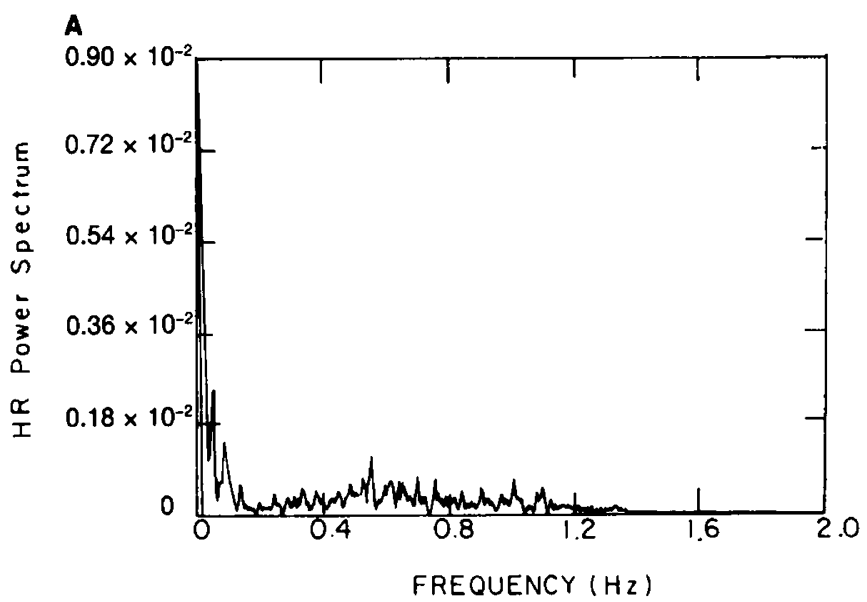

B

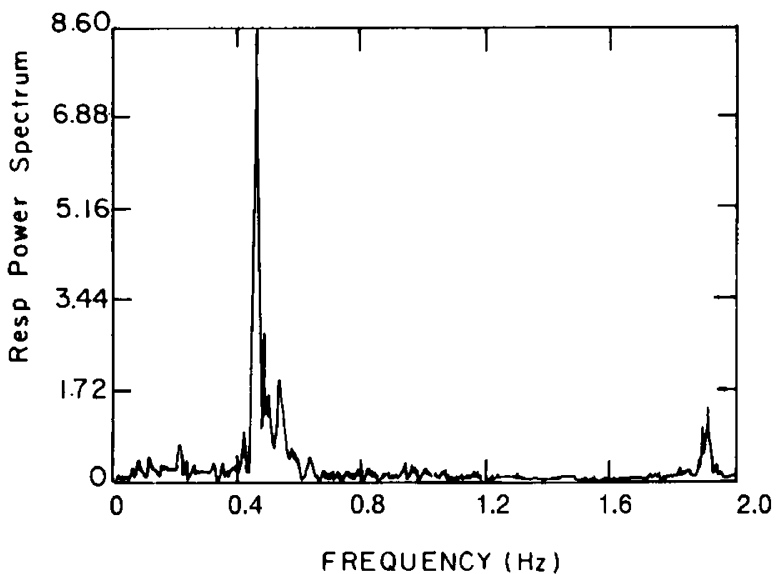

Figure 5. $A$, An HR power spectrum displaying a wide respiratory peak of the dispersive type. $B$, Corresponding respiratory power spectrum displaying a sharp peak at approximately $0.5 \mathrm{~Hz}$. 
$0.5-0.6 \mathrm{~Hz}$, meaning that the dispersion of the peak observed in the HRV spectrum is not necessarily caused by an unstable respiratory rhythm.

The second type of respiratory power spectra found in our study, the two-peak type, is also the one we found most commonly in premature neonates. Inspecting Figure $6 B$, we can easily define two frequency bands that contain most of the power in the respiratory power spectrum. The corresponding respiratory time series is shown in Figure 6A. The lower frequency band of respiratory activity is associated with the breathing bursts (13), or amplitude oscillations, and the higher one with the main breathing rate. The burst frequency is approximately an unstable $0.07 \mathrm{~Hz}(1 / \mathrm{T})$, and the respiration frequency is at $0.9 \mathrm{~Hz}$, each with its own harmonics. The maximal peak in the respiratory power spectrum occurs at the respiration frequency (f). Other peaks occur at $f \pm(n / T)$, where $\mathrm{n}$ is an integer and $\mathrm{T}$ is the period of the breathing bursts, estimated at $10-15 \mathrm{~s}$ from Figure $6 B$. The peak at the lowest frequency occurs at $1 / \mathrm{T}$, in this case approximately $0.07 \mathrm{~Hz}$. Many additional peaks occur at multiples of this frequency, in addition to the peaks centered around $\mathrm{f}=0.9 \mathrm{~Hz}$ and occurring at $0.07-\mathrm{Hz}$ intervals around $\mathrm{f}$.

In both breathing modes, for all the neonates investigated in this study (breathing spontaneously), the HR fluctuations series (256 s long) displayed a dispersed pattern of high-frequency power. However, because we did not have respiratory recordings for all the neonates, we were unable to correlate between neonatal age and the occurrence of either of the respiratory modes.
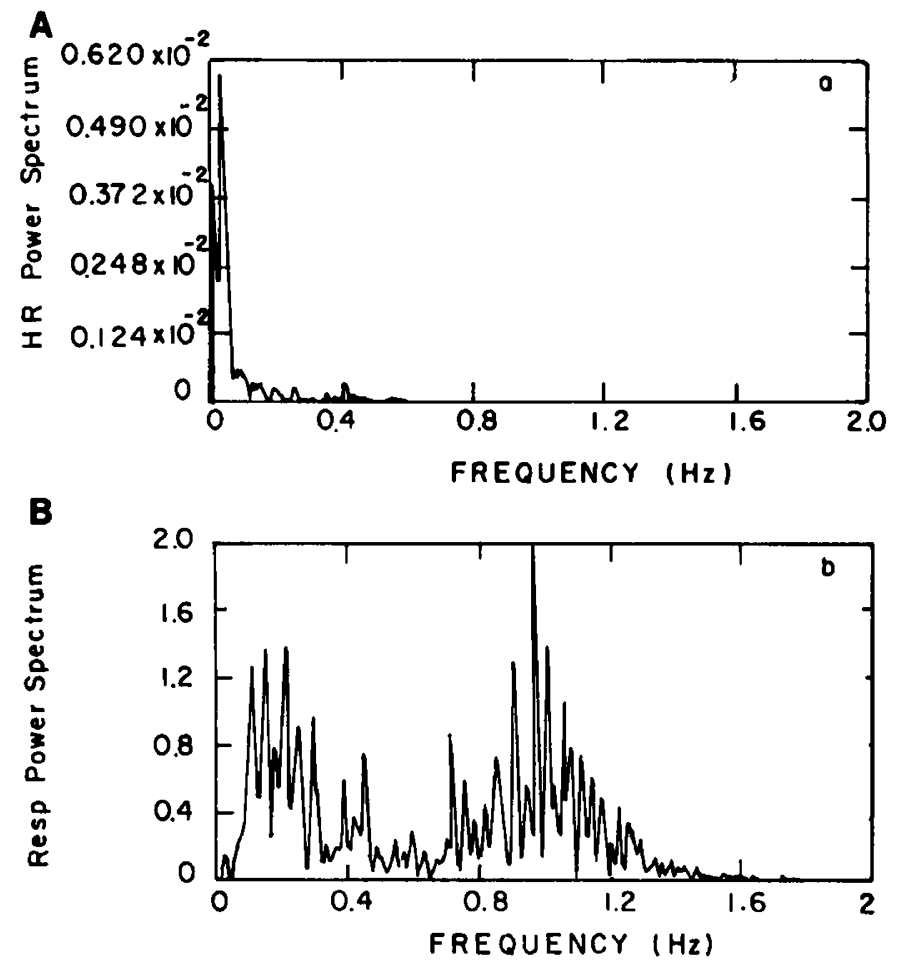

Figure 6. $A$, A respiratory time series displaying breathing bursts. $B$, Corresponding respiratory power spectrum displaying power primarily at two frequencies: the amplitude oscillation frequency at $0.07 \mathrm{~Hz}$ and the respiratory rate at $0.9 \mathrm{~Hz}$.
The power within the respiratory peak in HR power spectra in adults reflects the level of vagal tone, and its shape is usually a faithful replication of the respiratory peak in the respiration power spectrum. If respiration is regular, it appears as a narrow peak in the HR power spectrum; if it is irregular, it appears as a wide peak. What we observe in the neonates is that, even when the respiration is regular and the peak is narrow in the respiratory power spectrum, it may yet express itself in the HR power spectrum as a wide, dispersed respiratory peak. This could be one of the basic expressions of the difference between immature and mature autonomic control systems.

However, whatever the shape of the respiratory peak, its integral over the relevant frequency band in the HR power spectra still reflects the vagal tone. In particular, if the mean respiratory rate remains similar and only the width varies, then the value of the HFB integral still reflects the amount of vagal activity or maturation of vagal control.

\section{DISCUSSION}

During the last decade there has been an increasing interest in the beat-by-beat variation of HR generated by the ANS. Studies investigating the spectral content of HRV power in adults indicated the influence and interaction of three primary physiologic oscillations arising from respiration, blood pressure control or baroreceptor activity, and the vasomotor activity associated with temperature regulation (3-8). Although data from either preterm or full-term neonates are available, there still are many open questions regarding normal patterns for the HRV power spectrum (12-14). Total HRV power and its distribution as a function of frequency, as well as its evolution with neonatal maturation, require further investigation and have thus been approached in recent studies (11-13).

Dykes et al. (12) studied HRV and respiration in full-term infants and defined three spectral bands: $0-0.02 \mathrm{~Hz}, 0.02-0.2$ $\mathrm{Hz}$, and $0.2-2.0 \mathrm{~Hz}$. Our results confirm the adequacy of this band definition for neonatal HRV spectral analysis. In our study we discard the very low frequency band, $0-0.02 \mathrm{~Hz}$, to avoid possible inclusion of slow drift artifacts. The LFB, $0.02-0.2 \mathrm{~Hz}$, was subdivided into several narrow subranges, some of which are presented in Table 2.

The power distribution of HRV within the different frequency bands discloses two main features: first, the power spectra of HRV in neonates display considerable variation both within a given subject and across the population; and secondly, the ratio of the power in the LFB to that in the HFB seems to decrease with GA and thus in correlation with ANS maturation in infancy. The power ratio of these frequency bands has been assumed to reflect the sympathovagal balance (8).

Assuming that both increasing GA and increasing PA are accompanied by maturation of the newborn and its ANS, we observed a decrease in the values of the low- to high-power ratio with maturation. Figure $4 A$ displays the mean value of the low to high ratios taken from Table 2, showing a decreasing trend as a function of GA and PA. Comparing the mean low to high ratio of group A (24 h after birth) with group B (1-7 wk PA), both 33-35 wk CA, we notice a decline from a high ratio of 71 to a lower ratio of 34 as PA increases. This comparison 
was found to be statistically nonsignificant primarily because of the large SEM within the groups. Figure $4 B$ shows a similar trend found within group $B$ in the decreasing low to high ratio (as well as increasing high-frequency power and thus increasing parasympathetic predominance) as a function of PA.

Another comparison can be made between the combined $\mathrm{CD}$ group ( $\mathrm{CA}=39-41 \mathrm{wk})$ and $\mathrm{AB}$ group $(\mathrm{CA}=33-35 \mathrm{wk})$. Here we observed a significant $(p<0.01)$ decrease in both the low to high ratio and the mean HR as CA advanced. This result, again, probably reflects a developmental, relative increase in the parasympathetic contribution to HR control. This decrease in low to high ratio as a function of CA (Fig. 4A) is contributed by both the GA (comparing groups $A$ and $C$ ) and the PA (comparing groups A and B). These findings agree with previous reports showing that vagal tone increases as GA increases $(15,16)$. Our results are also consistent with studies on neonatal lambs $(9,10)$, which showed predominance of sympathetic over the parasympathetic activity in the fetus and in prematurity (if computed, these results would probably be expressed by a high low to high band power ratio in the HRV spectrum during the premature stage, as found in our study), with a pronounced increase of parasympathetic tone after birth.

The two independent respiratory modes $(17,18)$ found in the neonatal recordings in the present study point toward a major difference when compared with the single mode observed in the adult or mature respiratory recording. As mentioned, in all cases, the HR power spectrum did not show a clear and defined respiratory peak at the respiratory frequency but rather a wide, dispersive peak that probably resulted from an immaturity in the neural pathways (mainly parasympathetic) responsible for the translation of the respiratory activity into a nervous modulation of the instantaneous HR (or an immature transfer function from respiration to HR modulation) (19). As noted in the study by Giddens and Kitney (20) on full-term neonates, a strong association is found between HRV and BASA (breath amplitude variability). It seems that in the young preterm neonates the respiratory control system is based on a lowfrequency amplitude modulation mechanism system with repeated unordered switchovers to the mature type system, defined by a steady respiratory activity $(20,21)$. These switchovers are reminiscent of the sudden rises in autonomic activity related to the onset of breathing movements that we observed in the human fetus, when measuring fetal HR from an abdominal ECG signal and detecting respiratory movements by ultrasound imaging $(22,23)$.

In previous studies, the sleep state of the recorded neonates was found to affect both the respiratory and HR power spectra. Signorini et al. (14) reported a predominance of sympathetic activity during active sleep and vagal activation during quiet sleep. This group noted that the full-term newborn is found in active sleep $70 \%$ of the time. DeHaan et al. (24) found that quiet sleep is usually not observed in newborn infants before $35 \mathrm{wk} \mathrm{GA}$ and that the percentage of time during which quiet sleep occurs in newborn infants increases with GA. This observation may also, at least partly, explain or somewhat contribute to the significant difference in the low to high power ratio when comparing groups $\mathrm{AB}$ (33-35 $\mathrm{CA}$ ) and $\mathrm{CD}$ (fullterm infants). It is interesting to note that both the enhancement in vagal tone and the ability to experience quiet sleep may be reflections (dependent or independent) of autonomic maturation.

Kitney (25) proposed that an elevated low to high ratio might indicate increased risk for the infant. Baldzer et al. (11) observed that a difference in breathing rate affects both HRV and the low to high ratio; they divided the 20 full-term infants in their study into two groups according to the low to high ratio. They suggested that PA should be taken into consideration when defining risk factors as a function of low to high power. Because the low to high ratio is generally elevated in premature infants, our work emphasizes the need to account for the GA and PA of the neonate and preferably also to classify the respiratory mode and sleep state (quiet or active) of the infant before reliable risk factors can be determined (20-25).

Finally, we speculate that healthy maturation of the infant is reflected when the respiratory power spectrum in the preterm infant shows a decreasing amount of power at low frequency (breath amplitude modulation), a more narrow peak at mean breathing rate, or both and when the HR power spectrum displays a decrease in the low to high ratio. These conclusions are based on the assumption that a transition from a burst-like onset of breathing movements in the fetus to an irregular (amplitude- and frequency-modulated) respiration in the neonate to a regular, narrow-band breathing in a slightly older child reflects maturation. The observation of a decrease in mean HR and low to high ratio of power of HR fluctuations with progression of GA is another sign of development. Furthermore, our study suggests that parameters such as mean HR, and patterns of the HR power spectrum (in particular the low to high ratio) and of respiratory power spectrum (mainly width of respiratory peak and existence of low-frequency component) can all be used to provide insight into maturation and autonomic development of preterm and full-term neonates.

\section{REFERENCES}

1. Akselrod S, Gordon D, Madwed JB, Snidman NC, Shannon DC, Cohen RJ 1985 Hemodynamic regulation: investigated by spectral analysis. Am J Physiol 249:H867H875

2. Berger RD, Akselrod S, Gordon D, Cohen RJ 1986 An efficient algorithm for spectral analysis of $\mathrm{HR}$ variability. IEEE Trans Biomed Eng 48:305-307

3. Sayers BM 1973 Analysis of heart rate variability. Ergonomics 16:17-32

4. Hyndman BW, Kitney RI, Sayers BM 1971 Spontaneous rhythms in physiologic control systems. Nature 233:339-341

5. Kitney RI 1975 Entrainment of the human RR interval by thermal stimuli. J Physiol 252:37P-38P

6. Burton AC 1939 The range and variability of the blood flow in the human fingers and the vasomotor regulation of body temperature. Am J Physiol 127:437-453

7. Schweitzer A 1945 Rhythmical fluctuations of the arterial blood pressure. J Physiol 104:25P

8. Malliani A, Pagani M, Lombardi F, Cerutti S 1991 Cardiovascular neural regulation explored in the frequency domain. Circulation 84:482-492

9. Siimes ASI, Valimaki IAT, Antila KJ, Julkunen MKA, Metsala TH, Halkola LT, Sarajas HSS 1990 Regulation of HR variation by the autonomic nervous system in neonatal lambs. Pediatr Res 27:383-391

10. Assali NS, Brinkman CR, Woods R, Dandavino A, Nuwayhid B 1978 Ontogenesis of the autonomic control of cardiovascular functions in the sheep. In: Longo LD, Reneau DD (eds) Fetal and Newborn Cardiovasculary Physiology, vol 1. Garland STPM Press, New York, pp 47-91

11. Baldzer K, Dykes FD, Jones SA, Brogan M, Carrigan TA, Giddens DP 1989 HR variability analysis in full-term infants: spectral indices for study of neonatal cardiorespiratory control. Pediatr Res 26:188-195

12. Dykes FD, Ahmann PA, Baldzer K, Carrigan TA, Kitney R, Giddens DP 1986 Breath amplitude modulation of HR variability in normal full term neonates. Pediatr Res 20:301-308 
13. Nugent ST, Finley JP 1983 Spectral analysis of periodic and normal breathing in infants. IEEE Trans Biomed Eng 30:672-675

14. Signorini MG, Agostoni O, Brina A, Cerutti S, Di Michele R, Pagani M 1990 Spectral analysis of HR variability signals in newborns: physiological and clinical aspects. IEEE Comput Cardiol 1992:79-82

15. Porter F 1989 Pain in the newborn. Clin Perinatol 16:549-563

16. Fox NA 1983 Maturation of autonomic control in preterm infants. Dev Psychobiol $16: 495-504$

17. Kitney RI, Ong HG 1986 An analysis of cardiorespiratory control in babies and its relation to sudden infant death syndrome. Automedica 7:105-126

18. Aarimaa T, Valimaki IAT 1988 Spectral analysis of impedance respirogram in newborn infants. Biol Neonate 54:188-194

19. Berger RD, Saul JP, Cohen RJ 1989 Transfer function analysis of autonomic regulation: I. The canine animal rate response. Am J Physiol 25:H142-H152
20. Giddens DP, Kitney RI 1985 Neonatal HR variability and its relation to respiration. J Theor Biol 113:759-780

21. Aarimaa T, Oja R, Antila K, Valimaki I 1988 Interaction of $H R$ and respiration in newborn babies. Pediatr Res 24:745-750

22. Karin J, Hirsch M, Sagiv C, Akselrod S 1992 Fetal autonomic nervous system activity monitoring by spectral analysis of heartrate variations. IEEE Comput Cardiol 1992:479-482

23. Karin J, Hirsch M, Akselrod S 1993 An estimate of fetal autonomic state by spectral analysis of fetal heart rate fluctuations. Pediatr Res 34:134-137

24. DeHaan R, Patrick J, Chess FG, Jaco NT 1977 Definition of sleep state in the newborn infant by HR analysis. Am J Obstet Gynecol 127:753-758

25. Kitney RI 1984 New findings in the analysis of HR variability in infants. Automedica $5: 289-310$ 